INPLASY

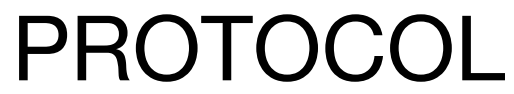

To cite: Xue et al. Effectiveness of respiratory muscle training for patients with obstructive sleep apnea: a protocol of systematic review and metaanalysis. Inplasy protocol 202040051. doi: 10.37766/inplasy2020.4.0051

Received: 10 April 2020

Published: 10 April 2020

Corresponding author: Shi-wei He

shiwei001he@aliyun.com

Author Affiliation:

Xi'an International Medical

Center Hospital

Support: SXKRDP

(2017SF-207)

Review Stage at time of this submission: The review has not yet started.

Conflicts of interest:

None.

\section{Effectiveness of respiratory muscle training for patients with obstructive sleep apnea: a protocol of systematic review and meta-analysis}

Xue, SMํ' Jia, J2; Fan, P3; He, SW4.

Review question / Objective: Is respiratory muscle training (RMT) effective and safety for patients with obstructive sleep apnea (OSA)?

Condition being studied: Obstructive sleep apnea, and respiratory muscle training.

Information sources: Electronic databases This study will involve searches of MEDLINE, EMBASE, Cochrane Library, CINAHL, Scopus, CBM, and CNKI. We will include any studies published from the inception to the present. No language restriction and publication status limitation will be imposed in this study. Other searches Further studies will be obtained from reference lists of associated studies, conference abstracts, and clinical trial registries.

INPLASY registration number: This protocol was registered with the International Platform of Registered Systematic Review and Meta-Analysis Protocols (INPLASY) on 10 April 2020 and was last updated on 10 April 2020 (registration number INPLASY202040051).

\section{INTRODUCTION}

Review question / Objective: Is respiratory muscle training (RMT) effective and safety for patients with obstructive sleep apnea (OSA)?

Condition being studied: Obstructive sleep apnea, and respiratory muscle training.

\section{METHODS}

Participant or population: There will be no limitation in participant characteristics in this study. All participants who were diagnosed as OSA will be included.

Intervention: In the experimental group, all patients must be treated with RMT. 
Comparator: In the control group, all patients can be treated by any interventions, except any forms of RMT.

Study designs to be included: All randomized controlled trials (RCTs) of RMT for the treatment of patients with OSA will be considered eligible.

Eligibility criteria: This study will include RCTs that compared the efficacy and safety of RMTI with other treatments for patients with OSA.

Information sources: Electronic databases This study will involve searches of MEDLINE, EMBASE, Cochrane Library, CINAHL, Scopus, CBM, and CNKI. We will include any studies published from the inception to the present. No language restriction and publication status limitation will be imposed in this study. Other searches Further studies will be obtained from reference lists of associated studies, conference abstracts, and clinical trial registries.

Main outcome(s): The primary outcome includes severity of OSA, as measured by polysomnography or any relevant tools. The secondary outcomes consist of hypopnea index, apnea index, respiratory event index, respiratory disturbance index, sleep-related quality of life, and any expected or unexpected adverse events.

Data management: Once the RCTs for inclusion have been identified, data outlined in the pre-designed standardized data extraction sheet will be undertaken from all eligible studies. Raw data will be collected from original RCTs by two researchers: first author, publication year, location, baseline characteristics of patients, diagnostic criteria for OSA, study setting, study design, study methods, intervention details in both experimental and control group, outcomes, and safety. Any discrepancies between two researchers will be solved by another researcher through discussion to reach a consensus. We will also contact primary authors if some unclear or insufficient information occurs.
Quality assessment / Risk of bias analysis: To evaluate the study quality of selected studies, we will use Cochrane Handbook for Systematic Reviews of Interventions tool based on seven items. The response for each one is reported as low, unclear or high risk of bias. Two researchers will independently assess the quality of RCTs. Any divergences will be solved through discussion to make decision with the help of another researcher.

Strategy of data synthesis: RevMan $\mathbf{5 . 3}$ software will be utilized for statistical analysis. We will pool the outcome results using risk ratio and $95 \%$ confidence intervals (Cls) for dichotomous data, and mean difference or standardized mean difference with $95 \%$ Cls for continuous data. Heterogeneity among included RCTs will be checked using I $^{2}$ statistic (I250\% shows high heterogeneity). We will pool the data across studies using a fixed-effects model if acceptable heterogeneity identified, and a random-effect model for obvious heterogeneity. Meta-analysis will be carried out if outcomes evaluated in the sufficient selected studies are comparable. In case of significant heterogeneity, subgroup analysis will be undertaken to check sources of obvious heterogeneity. Where appropriate we will also present narrative synthesis in a descriptive summary.

Subgroup analysis: To identify possible sources of obvious heterogeneity, we will carry out subgroup analysis in accordance with the study or patient characteristics, types of interventions, controls, and outcomes.

Sensibility analysis: To investigate the stability of study conclusions, we will undertake sensitivity analysis by excluding low quality studies.

Country(ies) involved: China.

Keywords: Obstructive sleep apnea; respiratory muscle training; effectiveness; safety. 